\title{
Problématique Maraîchère Induite Par La Nouvelle Dynamique Foncière Autour Des Bas-Fonds Urbains À Bouaké (Côte D'ivoire)
}

\author{
Kra Kouakou Valentin \\ Socio-économiste, Maître-assistant \\ Département d'Anthropologie et de Sociologie \\ Université Alassane Ouattara (Bouaké, Côte d'Ivoire)
}

Doi: 10.19044/esj.2018.v14n15p217 URL:http://dx.doi.org/10.19044/esj.2018.v14n15p217

\begin{abstract}
Urban agriculture in Côte d'Ivoire is an important activity in terms of food security, employment and response to environment. However in Bouaké, this agriculture and mainly gardening faces to a new land dynamics around intra-urban shallow. This situation results to emergence of new actors because of new stakes that represent shallows. As consequences we have a reduction of cultivated areas, an instability and a casualization of this type of agriculture. By analysis, it appears that of this sallow's upgrading leads a redefinition of operating rules of flooded fields, witch were dropped to gardeners. More, this public servitudes and unbuilt areas subject to illegal land commercial transaction and anarchic buildings. We deduce a modification of these spaces operating rules, problems of land government and a neglect of urban agriculture in public politics and city management in Côte d'Ivoire.
\end{abstract}

Keywords: Urban agriculture, sallow, Bouaké, land governance, urban gardening

Résumé

L'agriculture urbaine en Côte d'Ivoire constitue une activité importante en termes de sécurité alimentaire, d'emploi et de réponse aux problèmes environnementaux. Cependant à Bouaké, cette agriculture et principalement le maraîchage urbain fait face à une nouvelle dynamique foncière autour des bas-fonds intra-urbains. Cette situation est consécutive à l'émergence de nouveaux acteurs en raison des enjeux nouveaux que représentent les bas-fonds. Comme conséquences, nous avons une réduction des espaces cultivés, une instabilité et une précarisation de ce type d'agriculture. A l'analyse, il ressort qu'une revalorisation de ces bas-fonds a entrainé une redéfinition des règles d'exploitation de ces sols hydromorphes, 
jadis abandonnés aux seuls maraîchers. De plus, ces servitudes publiques, inconstructibles font l'objet de transactions foncières marchandes illégales et de constructions anarchiques. De la modification des règles d'exploitation de ces espaces, on en déduit des problèmes de gouvernance foncière et une négligence de l'agriculture urbaine dans les politiques publiques et dans la gestion des villes en Côte d'Ivoire.

Mots-clés : Agriculture urbaine, bas-fonds, Bouaké, gouvernance foncière, maraîchage urbain

\section{Introduction}

L'Afrique connaît une croissance démographique et une urbanisation rapides depuis la fin du $\mathrm{XX}^{\text {ème }}$ siècle. Dans ce contexte, les besoins alimentaires sont de plus en plus grandissants et le monde rural ne parvient plus à répondre efficacement à la question vivrière ; en raison des conditions bioclimatiques défavorables, l'exode rural et la problématique foncière. L'agriculture intra-urbaine et singulièrement le maraîchage se présente dès lors comme une alternative importante pour garantir la sécurité alimentaire. Ce type d'agriculture; (maraîchage intra-urbain) occupe une place primordiale dans la chaîne de valeur favorable à la nutrition. Sa proximité avec la ville, son accessibilité à toute période de l'année, ses réponses adaptées aux habitudes alimentaires induites par l'urbanisation et l'occidentalisation des populations africaines, font de ce maraîchage la plus importante chaine de valeur favorable à la nutrition.

Cependant, en Côte d'Ivoire et particulièrement à Bouaké, cette agriculture rencontre de nombreuses difficultés en rapport avec la situation foncière locale. De fait, les bas-fonds maraîchers de cette ville ancienne capitale de la rébellion font l'objet d'enjeux importants ces dernières années. Des recherches sur le terrain permettent de faire deux principaux constats.

Le premier constat tient de ce que sur la scène foncière à Bouaké, les bas-fonds qui jusqu'avant la crise militaro-politique de 2002 étaient essentiellement occupés par les producteurs maraîchers, font de plus en plus l'objet d'enjeux importants au sein d'une pluralité d'acteurs. La situation est perceptible à travers la multiplication du nombre d'agriculteurs, le retour des autochtones ainsi que l'intrusion de certains agents municipaux. Le second constat met en lumière que cette nouvelle dynamique foncière dans les basfonds intra-urbains a des impacts sur cette agriculture qui y est pratiquée. Dans ces conditions, cette activité, quoiqu'étant l'un des moteurs du développement local, reste instable est se précarise.

Ces constats suscitent des interrogations : Qu'est-ce qui explique cette émergence d'enjeux et d'acteurs nouveaux sur ces espaces ces dernières 
années ? Comment se manifeste cette instabilité et la précarisation du maraîchage intra-urbain à Bouaké ?

Il est question, à travers cette étude d'analyser la nouvelle dynamique foncière et la situation agricole en cours dans les bas-fonds maraîchers de la ville de Bouaké. De façon spécifique, il s'agit d'une part d'expliquer et comprendre l'émergence de nombreux acteurs sur ces espaces jadis abandonnés aux producteurs maraîchers et d'autre part d'analyser l'instabilité et le processus de précarisation du maraîchage intra-urbain à Bouaké.

\section{Méthodologie}

Cette étude a été menée en mars 2017 à Bouaké ; à travers les basfonds des quartiers Ahoungnansou, N'gatakro et Kôkô. La méthodologie adoptée s'appuie sur une approche qualitative menée; d'une part, auprès d'acteurs principaux que sont les producteurs maraîchers et les acquéreurs de lots dans les bas-fonds et autre part, auprès d'acteurs secondaires ; à savoir les populations autochtones, des agents municipaux et du Ministère de la Construction et de l'Urbanisme. A cet effet, la recherche documentaire, le guide d'entretien et l'observation directe ont été mobilisés pour recueillir les informations utiles à l'étude. Les informations prélevées sur le terrain ont fait l'objet d'une analyse du contenu pour en faire ressortir la substance utile à la compréhension de l'étude.

\section{Ancrage theorique}

L'étude s'appuie sur la théorie de l'acteur stratégique (CROZIER et FRIEDBERG, 1977) et la théorie du choix rationnel (BOUDON, 2002). La théorie de l'acteur stratégique permet de mettre en exergue l'acteur qui ne se laisse jamais totalement contraindre par le système mais dispose des marges de manœuvre sur lesquelles il joue pour atteindre ses objectifs individuels. Dès lors, nous avons pu comprendre comment face au "régime militaire' et ensuite à l'administration officiel, certains acteurs parviennent à contourner les normes pour s'imposer et contrôler le foncier dans les bas-fonds maraîchers de la ville de Bouaké. Quant à la théorie du choix rationnel, elle stipule que face à plusieurs options, l'individu opère des choix en tenant compte des avantages, des risques. Elle nous permet de comprendre la négligence des différents acteurs vis-à-vis du l'agriculture urbaine.

\section{Résultats}

\section{De la singularité à la pluralité des acteurs fonciers des bas-fonds intra urbain}

La dynamique foncière autour des bas-fonds intra-urbains à Bouaké se perçoit à travers la pluralité des acteurs qui y interagissent. 


\section{Les producteurs de maraîchers}

Les premiers acteurs à véritablement exploiter les bas-fonds intraurbains à Bouaké furent les maraîchers. Ces espaces laissés en friche ; par les autochtones $^{5}$ et les pouvoirs publics ${ }^{6}$ sont occupés par ces maraîchers depuis les années 1980. Il s'agissait de quelques personnes disséminées sur ces espaces jadis qualifiés de 'terrains sans valeurs'. Les propos de $\mathrm{YEO}^{7}$ traduisent clairement la taille de la population qui s'intéressait à ces espaces : «Avant, on était un ici. Il y avait beaucoup de places mais on dépassait pas cinq personnes. C'est aujourd'hui tu peux voir les gens ici comme ça».

Ces premiers maraîchers étaient essentiellement issus des communautés du Nord de la Côte d'Ivoire (Sénoufo et Malinké) ainsi que ceux de pays limitrophes (Burkinabés, Maliens). Ils s'y sont installés dans le but de pratiquer maraîchage. L'exploitation de ces espaces hydromorphes bénéficie de leurs expériences antérieurement acquises dans leurs régions d'origine dans l'activité maraîchère. C'est d'ailleurs ce que confirment les propos de TRAORE ${ }^{8}$ : "Moi, je suis venu ici en 1989. Je ne travaillais pas donc je suis venu me débrouiller dans jardinage ici. C'est ce que je faisais au Mali. J'ai commencé jardinage là chez nous. On faisait oignon, chou, carotte. C'est mon premier travail ».

Dans certains cas, en plus du maraîchage, des agriculteurs ont profité pour construire des maisons dans le but de se rapprocher de leur lieu de travail. Le facteur proximité avec le lieu d'exercice de l'activité a véritablement contribué à la construction des toutes premières habitations dans les bas-fonds. L'expérience de YEO est illustrative : "C'est mon grand frère qui faisait champ ici. Il avait demandé la place aux Baoulé. Moi je suis venu en 1984 et comme là où on habitait à Sokoura était loin d'ici, on a construit petite maison ici pour habiter. Tu vois tous ceux qui ont maison et leur famille ici dans basfond là, c'est comme ça ils sont venus ».

Retenons pour l'essentiel que les populations allochtones et allogènes furent les premiers maraîchers à s'installer sur les bas-fonds jadis négligés et abandonnées par les autochtones. Cependant, depuis quelques années, l'on observe une évolution quantitative de ces maraîchers ainsi que l'émergence de "'nouveaux acteurs".

${ }^{5}$ Les autochtones baoulé n'exploitent pas les bas-fonds parce qu'impropres à culture de l'igname ; leur aliment de base.

${ }^{6}$ Pour les pouvoirs publics (Etat et municipalités), les bas-fonds constituent des servitudes et des espaces non constructibles.

${ }^{7}$ YEO est un maraîcher d'ethnie sénoufo rencontré dans le bas-fond reliant les quartiers Ahougnansou et N'gatakro lors de nos enquêtes de mars 2017.

${ }^{8}$ TRAORE est un maraîcher d'origine malienne, rencontré dans le bas-fond du quartier Koko, lors de nos enquêtes de mars 2017. 


\section{Du retour des autochtones}

Les autochtones baoulés sont les premiers à occuper la région de Bouaké dès leur arrivée de la Gold Coast (actuel Ghana) au XVII ${ }^{\text {ème }}$ siècle. Ce peuple agriculteur s'adonnait principalement à la culture de l'igname qui demeure sa nourriture de base. Les bas-fonds n'étant pas propices à cette culture, ils n'étaient pas considérés comme des terres arables. Cela ne signifie pourtant pas que les bas-fonds sont sans importance en pays baoulé. Bien au contraire, ils constituent des zones de chasse, des réserves d'eau douce et bien plus des lieux sacrés (rivières et bois sacrés). Les cultiver serait en quelque sorte indisposer les génies protecteurs.

Avec l'urbanisation, ces espaces perdent de leur importance socioculturelle. Les autochtones baoulés, plutôt que d'y laisser pousser la broussaille, qui pourrait être des nids de bandits et autres serpents, les cèdent aux premiers allochtones et allogènes intéressés; sans contrepartie. Ces derniers selon les relations qu'ils entretiennent avec leurs tuteurs baoulés, peuvent leur donner quelques produits de la récolte. $\mathrm{KOFFI}^{9}$ l'exprime à travers ses propos: "Ce sont nos parents qui ont donné les terrains aux Sénoufo que vous voyez. Avant, il y avait beaucoup de serpents et de scorpions qui étaient dans les herbes du bas-fond et ils sortaient pour venir dans les cours. Quand les gens nettoient pour faire champ au moins ça chasse ces bêtes. Les Senoufo aussi, quand ils récoltent leur riz ou leur choux, y a d'autres qui sont reconnaissants, ils viennent donner un peu aux vieux».

Disons que durant les premières décennies de l'urbanisation et donc en période de disponibilité foncière, les bas-fonds avaient perdu de leur importance aux yeux des autochtones. Non seulement ils ont été désacralisés, impropres à la culture de l'igname mais surtout inconstructibles et donc abandonnés. Cependant ces dernières années, l'on observe des mutations, une volte-face ou un retour des autochtones sur ces portions de terres à l'intérieur de la ville. Cette situation s'explique par le fait qu'aujourd'hui, leurs villages sont phagocytés par la ville de Bouaké et les terres coutumières sont presque totalement expropriées pour cause d'utilité publique. Cette situation est difficilement vécue par ces autochtones pour qui la purge des droits coutumiers n'a pas été correctement ou pas du tout faite. Les propos du chef $\mathrm{du}$ village de Koliakro $^{10}$ traduisent clairement ce que vivent ces populations : "Toutes nos terres sur lesquelles on faisait nos champs ont été prises et on a rien reçu en retour. On est obligé d'aller demander des parcelles auprès de nos frères d'Akanzakro ${ }^{11}$ pour faire nos champs. Aujourd'hui la

${ }^{9}$ KOFFI est un jeune autochtone baoulé interrogé au bas-fond d'Ahougnansou lors de nos enquêtes de 4 mars 2017.

${ }^{10}$ Village autochtone phagocyté par le quartier Ahougnansou SICOGI.

${ }^{11}$ Akanzakro est un village situé à 5 kilomètres sur le tronçon Bouaké-Sakassou. 
seule terre qui me reste c'est ce cimetière que vous voyez (il l'indique du doigt)».

Dans ce contexte de raréfaction de terres, certains autochtones se rabattent sur les parcelles de bas-fonds qui restent leurs seules réserves foncières. Le retour des autochtones ne se fait pas à l'effet d'y pratiquer l'agriculture mais bien plus pour la vente de terrain après des lotissements villageois. Ainsi, le bas-fond reliant Ahougnansou à Ngatakro et celui entre Ngatakro et Houphouetville ont été lotis. De nombreuses constructions de maisons y sont en cours et remplacent progressivement les cultures maraîchères.

L'intérêt porté par les autochtones baoulés sur les bas-fonds ces dernières années est consécutif à la raréfaction des terres coutumières en raison de l'urbanisation. Ces espaces inondés restent pour eux, les seules réserves foncières pouvant faire l'objet de marchandisation.

\section{De l'intrusion de certains agents municipaux}

Les pouvoirs publics dont la Mairie, ont la gestion des espaces non viabilisés ou non construits que sont entre autres les servitudes, les bas-fonds. Tous ces espaces font partie du domaine public. C'est à titre que la Collectivité Territoriale (la Mairie) assure la gestion de ce domaine ; conformément à l'article 4 du décret $\mathrm{n}^{\circ} 2005-261$ du 21 juillet $2005^{12}$. Ce décret stipule que cette dernière "peut notamment, dans le cadre de la gestion de son domaine public, accorder des autorisations d'occupation temporaire, à titre précaire et révocable, gratuit et onéreux, sur les terrains non occupés de ce domaine ». Ces autorisations d'occupation se font donc en tenant compte des dispositions en vigueur ; surtout quand elles le sont à titre onéreux. Une taxe forfaitaire est instaurée à cet effet et légalement perçue par la municipalité. Or sur le terrain, l'application des dispositions légales est biaisée par certains agents municipaux qui usent de pratiques informelles, illicites pour soutirer de l'argent à certains maraîchers. Ce fait a été signalé par les agriculteurs du basfond de KôKô : "(...) nous on n'a pas assez de problème ici. Quand les gens de Mairie viennent là, on s'arrange souvent pour leur donner dix mille (10000) francs, souvent même rien (petit sourire...) et on met ça dans plaisanterie en le suppliant de prier pour que le marché soit bon pour nous.(...) On leur offre souvent des boules de tomates, carottes pour leur famille. Et cela nous réconcilient tous davantage » $\left(\right.$ Mariam $\left.^{13}\right)$.

12 Décret $\mathrm{n}^{\circ} 2005-261$ du 21 juillet 2005 fixant les modalités d'application en matière d'Urbanisme et d'Habitat, République de Côte d'Ivoire.

${ }^{13}$ Productrice de maraîcher, d'ethnie malinké ; interviewée au bas-fond de Kôkô, le 22 février 2017. 
"Quand ça marche bien. On cotise souvent 2500 à 5000 chacun pour leur donner », renchérit Aminata ${ }^{14}$.

A la question de savoir s'ils reçoivent des reçus attestant qu'ils ont effectivement versé de l'argent aux agents de la Mairie ; à titre d'occupation du domaine public, $100 \%$ des maraîchers interrogés dans la zone de Kôkô ont répondu '"non". "Ils (agents de la mairie) disent que c'est arrangement entre nous. Si on veut reçu là ; on va payer beaucoup d'argent hein. Nous-mêmes on connait ça donc on demande pas reçu.» (Mariam).

En ce qui concerne le bas-fond reliant Ahougnansou à Ngatakro et celui de Ngatakro - Houphouetville, l'agriculture y est de plus en plus menacée par les lotissements et les constructions de maisons. Il n'est pas signalé d'intervention directe des agents municipaux par les agriculteurs. Cependant, certains acquéreurs de lots rencontrés sur le terrain citent formellement la Mairie comme étant un des acteurs des transactions foncières qui y ont cours. Des propos l'attestent bien : "Lot là c'est pour mon fils. Il a payé avec les chefs de kplikro ${ }^{15}$. La Mairie aussi est informée et elle a donné son accord. Lot d'ici là, c'est pas bon comme les autres lots donc c'est business on fait » (Maïmouna $\left.{ }^{16}\right)$.

«Affaire de Mairie là, tu sais, on peut pas construire maison sans eux. Donc quand c'est comme ça, il faut traiter avec eux. Quand on achète le lot avec les baoulés, on va aussi à la Mairie pour faire papier là bas. Souvent Mairie vient mettre croix rouge et écrit A.D. sur la construction. Entre nous ici, on lit ça “'A Discuter'” au lieu de A Démolir. (Rires..)». (OUATTARA ${ }^{17}$ ).

Du côté de la Mairie, des gardes municipaux et le bureau de domaines ont été contactés. Les différents agents interviewés disent ne pas être informés des sommes versées par certains agriculteurs et encore moins des lotissements et transactions foncières en cours dans des bas-fonds de la ville. Ils décrient d'ailleurs l'illégalité de telles actions et fustigent les agents véreux qui se prêteraient à ce type de pratiques.

Les différents propos recueillis indiquent que des agents municipaux interviennent clandestine sur le terrain pour soutirer de l'argent aux agriculteurs ou encore pour bénéficier d'une contrepartie liée aux acquisitions de lots dans les bas-fonds.

\footnotetext{
${ }^{14}$ Producteur de maraîcher, d'ethnie senoufo ; interviewée au bas-fond de Kôkô, le 22 février 2017.

${ }^{15}$ Village autochtone phagocyté par le quartier Ahougnansou.

${ }^{16}$ Représentante d'un acquéreur de lot dans le bas-fond Ahougnansou-Ngatakro ; interviewée le 3 mars 2017.

${ }^{17}$ Acquéreur de lot dans le bas-fond Ahougnansou - Ngatakro, interviewé le 3 mars 2017.
} 


\section{Impacts de cette dynamique foncière sur le développement du maraîchage}

Les bas-fonds de la ville de Bouaké représentent des enjeux nouveaux pour les différents acteurs. Ces enjeux constituent une menace pour le maraîchage qui du coup voit ses espaces réduits et se précarise.

\section{Réduction des espaces cultivés}

L'un des impacts de la nouvelle dynamique foncière sur le maraîchage urbain se présente en termes de réduction des espaces cultivés par maraîcher. Deux facteurs essentiels expliquent cette situation: la croissance démographique des maraîchers et l'urbanisation des bas-fonds.

Il convient de noter qu'avant la crise militaro-politique en septembre 2002, le maraîchage intra-urbain à Bouaké était dominé par quelques acteurs disséminés sur les bas-fonds à l'intérieur de la ville. Il y avait donc une abondance foncière pour une faible population de maraîchers. Cependant, depuis 2002 l'on observe l'arrivée de nouveaux maraîchers dans ces espaces inondés. Nous ne disposons pas des statistiques formelles mais nos enquêtes et les témoignages de certains interviewés attestent de cette évolution : "Il y avait beaucoup de places mais on ne dépassait pas cinq personnes (...). Mais depuis gbangban ${ }^{18}$ là, les gens sont devenus beaucoup ici. On avait tous peur d'aller loin dans les champs qui sont en brousse. On peut te tuer si tu vas en brousse pour faire champ. Les gens laissent les champs en brousse pour venir voir nous les premiers et on leur donne un peu de terrain pour faire champ. Si y a des coins que nous on travaillait pas, les nouveaux viennent dessus et même à côté aussi pour faire champ. Je peux dire qu'on est devenu dix fois plus beaucoup qu'avant. » (YEO).

Mariam renchérit : "Mon mari et son ami étaient seuls ici. On a des frères qui sont venus et on leur a donné petite place. Eux, ils disent que leur champ est en brousse donc ils ne peuvent plus partir là bas à cause de gbangban là. Sinon avant y avait deux (2) personnes mais aujourd'hui on peut compter huit (8) ou dix (10) personnes comme ça ».

En plus des témoignages, ce qui est frappant à l'observation, c'est surtout l'occupation totale des bas-fonds, la densité de l'activité agricole et la forte présence humaine. En plus des maraîchers, l'on y rencontre des éleveurs, des commerçants de produits phytosanitaires et des acheteurs bord champ.

Le second facteur de réduction des espaces agricoles est l'urbanisation des bas-fonds. En effet, la croissance démographique rime avec un besoin en logements urbains. Dans ces conditions, les terres inondées laissées en friches sont de plus en plus convoitées. Les populations autochtones ayant perdu

\footnotetext{
${ }^{18}$ Néologisme ivoirien pour désigner la crise militaro-politique de 2002 à 2011.
} 
toutes leurs terres au profit de l'urbanisation, reviennent dans les bas-fonds qu'ils avaient autrefois abandonnés. Il s'agit pour eux de les lotir et de procéder à des ventes de ces terres qui constituent leurs seules réserves foncières. Sur le terrain, l'on constate le lotissement des bas-fonds (présence de bornes) et la construction de nombreuses maisons.

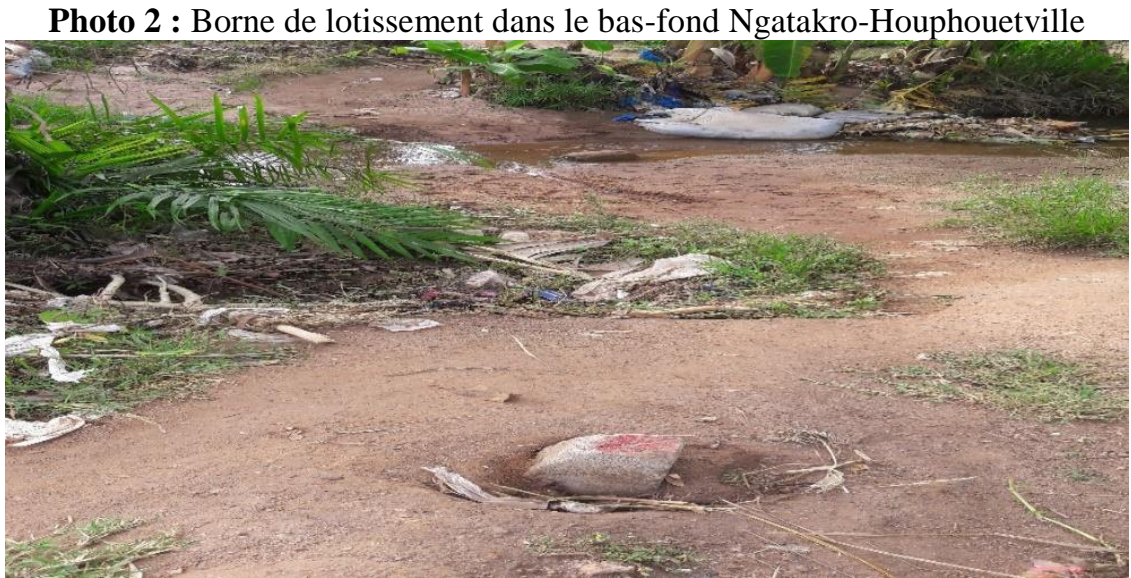

Source : KRA K. Valentin, mars 2017.

La conséquence de telles actions c'est que ces terres sont de plus en plus réduites et remplacées par les constructions. Les propos de YEO sont éloquents à ce sujet: "Les Baoulé ont loti tout les bas-fond. Tu vois, les maisons là ? Je faisais champ là bas. Quand tu passais pour venir ici, tu as vu les bornes là? Tu as vu les gens qui sont en train de faire fondation de maison dans mon champ de riz là? Demain je ne vais plus avoir place pour faire champ. Ça me fait mal mais c'est terre des Baoulé. Ils font ce qu'ils veulent ».

En somme, l'on enregistre une augmentation du nombre des maraîchers ainsi que de nombreux acquéreurs de lots dans les bas-fonds à l'intérieur de la ville de Bouaké. Cette situation est consécutive à la valeur marchande des produits maraîchers, à la question du chômage mais également à la crise militaro-politique qui continue de marquer cette ville. De plus, de nombreux maraîchers sont menacés par l'urbanisation anarchique des basfonds intra-urbains.

\section{De l'instabilité et la précarisation du maraîchage périurbain à Bouaké}

La problématique foncière en cours dans les bas-fonds à l'intérieur de la ville de Bouaké a un impact sur le maraîchage. De fait, cette instabilité est consécutive à la pression qu'exerce l'urbanisation sur les terres agricoles intraurbaines notamment les bas-fonds. De plus en plus, ces espaces sont vendus et construits au détriment du maraîchage. Dans ces conditions, les maraîchers sont dans une position de faiblesse, de vulnérabilité; d'autant plus qu'ils ne disposent d'aucun titre foncier ou tout document administratif pouvant leur 
servir d'argument pour s'opposer à cette transformation des bas-fonds en terrains battis. Tout donne l'impression qu'ils sont traqués de toute part par les acquéreurs de terrain comme l'expriment bien certains maraîchers : "Il n'y a longtemps je fais choux et salade ici. Mais depuis dix (10) ans ou bien six (6) ans, on voit les gens viennent construire maison ici. Regarde maison d'à côté, avec l'autre qu'ils sont en train la fait là. Quand c'est comme ça, nous même on ne comprend rien. C'est comme si on nous chasse partout. Si je coupe choux là, ce n'est pas sûr que je gagne place là encore pour planter choux » (Salam) ${ }^{19}$.

«Nous même là, on ne sait pas si on doit faire champ encore (pause), parce que terrain là est loti. Tu peux faire champ et puis une semaine après quelqu'un vient pour construire dedans. Ça te fait mal mais tu ne peux rien dire. Nous on est là en attendant. C'est comme ça et on ne sait pas où on va partir maintenant » (YEO).

A travers ces propos, l'on perçoit l'impuissance des maraîchers face à l'avancée de l'urbanisation qui à terme phagocytera tous les bas-fonds. Audelà des constructions, il faut faire cas d'autres activités anthropiques liées à l'urbanisation. C'est l'exemple des ordures ménagères souvent déversées dans les bas-fonds et qui entravent le développement de l'agriculture urbaine. Les maraîchers rencontrés ont tous souligné la nuisance qu'elles représentent pour leurs cultures mais également pour les terres cultivables. Or à Bouaké, les basfonds donnent l'impression d'être des décharges à ordures incontrôlées. Les ordures déversées s'accumulent et occupent progressivement de grands espaces de bas-fonds agricoles. De plus les sachets plastiques qu'elles contiennent ont une incidence négative sur la fertilité des sols.

Photo 3 : Ordures ménagères dans le bas-fond Ngatakro - Houphouetville

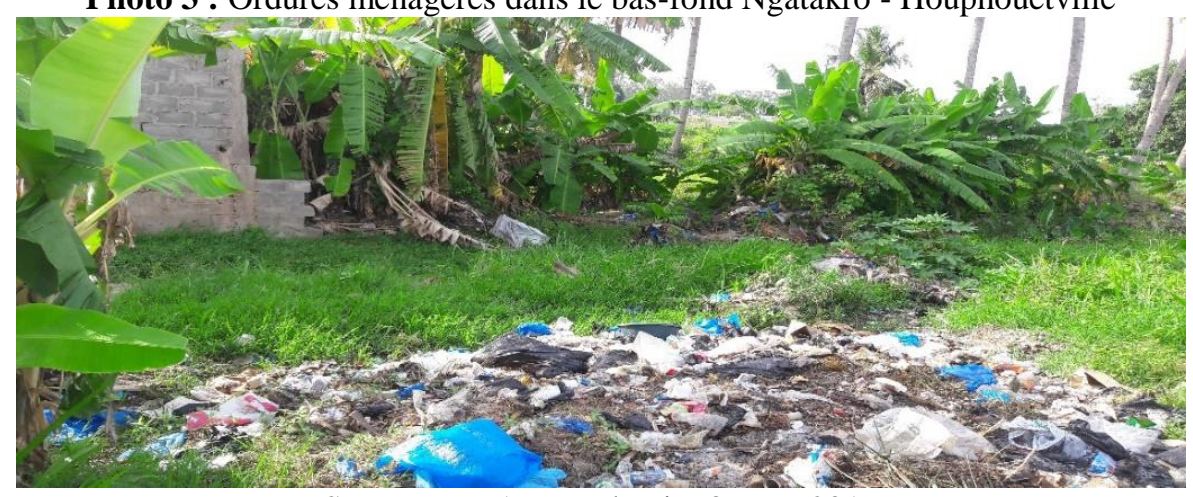

Source : KRA K. Valentin, 3 mars 2017

Les bas fonds à l'intérieur de la ville de Bouaké sont soumis à une forte pression de l'urbanisation. Il s'en suit une insécurité foncière qui fait du maraîchage une activité instable et précaire.

\footnotetext{
${ }^{19}$ Maraîcher du bas-fond de Dar-es-salam, interviewé le 6 mars 2017.
} 


\section{Discussion}

De la revalorisation à la redéfinition des règles d'exploitation des basfonds intra-urbains

La pression foncière est l'une des conséquences induites par l'urbanisation et la croissance démographique de la ville de Bouaké ces dernières années ; avec le retour massif des déplacés de guerre et des différents services (publics et privés). Dans ces conditions, l'accès au foncier pour le logement et même l'agriculture devient une problématique importante que les différents acteurs tentent de résoudre en développant des stratégies individuelles ou collectives pour s'imposer les uns aux autres. C'est en cela qu'intervient la théorie de l'acteur stratégique.

En effet, le nœud gordien de la question foncière urbaine à Bouaké et notamment dans les bas-fonds intra-urbains, c'est avant tout la multiplicité des acteurs ces dernières années. Si quelques décennies au paravent (jusqu'en 2002), ces espaces étaient négligés et laissés aux mains de quelques maraîchers, aujourd'hui ils font l'objet d'une revalorisation. Cela se perçoit à travers l'émergence d'une pluralité d'acteurs et une course effrénée pour l'acquisition de ces bas-fonds. Leur valeur d'usage (pour l'agriculture) fait place progressivement à leur valeur marchande. C'est d'ailleurs cette dernière valeur qui explique le retour en force des populations autochtones dans la gestion de ces portions de terres qu'ils avaient oubliées, voire abandonnées aux allochtones et allogènes durant les périodes d'abondance foncière. Le retour des propriétaires fonciers coutumiers et l'attribution de terrains lotis dans les bas-fonds à de nouveaux acquéreurs, instaurent une situation concurrentielle et même une situation de domination ou d'exclusion des maraîchers. Selon FLEURY et DONNADIEU (1997 : p. 46) « La concurrence pour le foncier est donc une caractéristique à part entière de l'agriculture urbaine d'autant que la valeur foncière des terrains est en générale supérieur à la seule prise en compte des potentialités agricoles ». A cet effet, le capital social et le capital financier ont été mobilisés par les autres acteurs pour s'imposer aux maraîchers présentés comme le maillon faible de la chaîne. D'ailleurs dans cette concurrence foncière, les espaces agricoles restent comparativement moins valorisés par rapport à ceux consacrés à l'immobilier. Ainsi les propriétaires coutumiers, en se basant sur leur capital social (statut d'autochtonie) peuvent réguler le jeu foncier. C'est en cela que CHOUAIBOUB et SINKONDO (2002 : p. 26) affirment que «les droits sur le sol se définissent par rapport à l'appartenance à un groupe social et en fonction d'un droit traditionnel ».

Quant aux acquéreurs de lots, il s'agit de capter la ressource foncière en faisant intervenir leur capital économique (ou les moyens financiers). Le capital économique est le plus mobilisé par les allochtones et les allogènes dans le jeu foncier. Dans ces conditions, il corrompt la gestion ou la conception 
coutumière des terres en pays baoulé. De fait, pour le peuple baoulé, la terre (Assiê) est sacrée, inaliénable et ne peut faire l'objet de transaction marchande. Or, dans un monde monétarisé, cette conception coutumière devient caduque et le capital économique s'impose aux autres types de capitaux. C'est d'ailleurs ce qui pousse les autochtones et certains agents de la municipalité à redéfinir leur position en ce qui concerne l'exploitation des bas-fonds intraurbains. Ces espaces deviennent de véritables lieux de marchandisation où les moins nantis financièrement comme les maraîchers sont de plus en plus exclus. Cette situation corrobore l'idée de MASSIAH et TRIBILLON (1988 : p. 161) selon laquelle; "le foncier est encore un moyen d'enrichissement ». La course à l'enrichissement est donc un déterminant important qui vient modifier ou redéfinir les règles d'accès et d'exploitation des bas-fonds à l'intérieur de la ville. Il s'agit d'un enrichissement pour les autochtones et des agents de la municipalité mais également pour les acquéreurs dans la mesure où posséder une portion de terre est synonyme de capitalisation et d'aisance financière.

La revalorisation des bas-fonds intra-urbains à Bouaké a pour conséquences, le recul du maraîchage, l'exclusion des paysans en raison de nouvelles règles d'exploitation qui privilégient le capital économique (ou financier). Ce constat confirme l'idée selon laquelle "la ville a un coût et que faute de pouvoir l'assumer, les pauvres doivent céder la place» (HESSELING, 1991, 148). Il s'instaure dès lors une dynamique foncière faite d'inégalités socio-économiques dans l'accès à ces espaces urbains. Par ailleurs, du lotissement et de la marchandisation de ces bas-fonds, se dégage la problématique de la gouvernance foncière dans la localité.

\section{La problématique de la gouvernance foncière}

L'une des problématiques auxquelles sont confrontées les villes ivoiriennes depuis la dernière décennie $\mathrm{du} \mathrm{XX}^{\mathrm{ème}}$ siècle est la gouvernance foncière. A l'analyse, deux principaux facteurs peuvent être évoqués : la crise militaro-politique de 2002 à 2011 et les faiblesses de la politique de décentralisation en cours dans notre pays.

A Bouaké, la question foncière reste difficile à gérer en raison des dix années de crise militaro-politique qu'a connue le pays et qui fut de cette ville la capitale de la rébellion. Durant cette période où l'administration officielle était absente dans les zones Centre, Nord et Ouest (CNO), ce fut une véritable anarchie au niveau de la gestion foncière locale. De nombreux lotissements illégaux dont ceux des bas-fonds maraîchers avaient eu lieu. Ce désordre foncier persiste et s'exacerbe; même avec le retour de l'administration dans ces zones antérieurement occupées par la rébellion. La conscience collective des populations locales et principalement celles de Bouaké reste encore durablement marquée et s'inscrit dans une logique de 
continuité de la crise militaro-politique. Tout se passe comme si l'administration officielle était absente. C'est donc dans ce contexte encore trouble, que certains acteurs ; notamment les populations autochtones profitent pour procéder à des transactions foncières et des lotissements entachés d'irrégularité. Les seules réserves foncières à l'intérieur de la ville sur lesquelles ont lieu ces lotissements irréguliers sont en général les espaces hydromorphes et non constructibles des bas-fonds. Or en réalité, ces bas-fonds intra-urbains sont frappés de servitudes d'urbanisme par l'Etat ivoirien.

Au-delà de la crise militaro-politique comme facteur de ce "désordre foncier' dans la ville de Bouaké, il faut aussi faire cas des insuffisances liées à la politique de décentralisation en cours dans notre pays depuis la fin du $\mathrm{XX}^{\text {ème }}$ siècle. En effet, cette politique se présente comme un moyen de promotion de la démocratie et le développement local fondée sur la participation active des populations. Ainsi, en ce qui concerne la gestion foncière, émergent de nombreux acteurs que sont les collectivités territoriales, des acteurs privés, les populations ; à côté de l'Etat. On passe donc d'une situation d'omniprésence étatique à une situation de " moins d'Etat» (LAURENT, 2000 : p. 3). Or avec ce moins d'Etat ou le recul de sa capacité coercitive, s'installe un certain laxisme administratif qui conduit l'incivisme, à l'élaboration de petits gouvernements locaux ou à une sorte "d'ailleurs dans le dedans », selon LARENT (ibid : p. 3). C'est dans cette atmosphère faite de nombreux acteurs, d'anarchie et de pluralité juridique (droits coutumiers et droit moderne) que les bas-fonds maraîchers font l'objet de réappropriation et lotissements par les autochtones ainsi que de constructions illégales par les acquéreurs. A cet effet, les populations autochtones et certains agents de la municipalité s'imposent comme les véritables gestionnaires des bas-fonds de la ville. Pour les autochtones, il s'agit de capter rapidement les dernières réserves foncières urbaines ou encore de rattraper le manque à gagner des maigres compensations issues de la purge des droits fonciers coutumiers versées par les pouvoirs publics. Quant aux agents municipaux, il est question de profiter du laxisme de l'administration publique pour se constituer d'importantes ressources financières.

La nouvelle dynamique foncière en cours dans les bas-fonds intraurbains à Bouaké dénote des insuffisances de la gouvernance foncière locale. Au delà de cette question relative à la gouvernance foncière locale, il faut également mentionner la négligence dont fait l'objet le maraîchage urbain dans les politiques publiques et de gestion des villes en Côte d'Ivoire.

\section{Le maraîchage urbain : un secteur négligé dans les politiques de gestion des villes}

L'agriculture urbaine et principalement le maraîchage intra-urbain est une activité de première importance en Côte d'Ivoire. Elle se présente non 
seulement comme un secteur pourvoyeur d'emploi mais contribue efficacement à la sécurité alimentaire. «Sa proximité avec la ville, son accessibilité à toute période de l'année, ses réponses adaptées aux habitudes alimentaires induites par l'urbanisation et l'occidentalisation des populations africaines font de ce type de maraîchage la plus importante chaine de valeur favorable à la nutrition »(KRA, 2017). Malgré tout, il ne fait pas partie des choix ou des priorités des pouvoirs publics, des collectivités territoriales et des opérateurs économiques car étant perçu comme moins stratégique et moins rentable. C'est ici qu'intervient la théorie du choix rationnel pour la compréhension des options des différents acteurs vis-à-vis de l'agriculture urbaine.

A travers le monde, et surtout dans les pays développés, l'importance de l'agriculture urbaine est avérée ; en raison de sa multifonctionnalité. Vus ses enjeux pour les différents acteurs urbains, les politiques publiques dans ces pays développés en tiennent nécessairement compte dans l'aménagement urbain. Bien plus, elle est définie comme « un outil durable de l'aménagement urbain » auquel elle est même inclue dans cette pays comme au Canada (Anne-Cécile Daniel, 2013, p50). En Europe, 1'exemple de la France où le Conseil Général de l'Île de France, les Conseils Généraux des Yvelines et l'Essonne ont mis en place des politiques en faveur de l'agriculture urbaine (BLAUDIN D. T. C. et al, 2009) est édifiant. Dans certains en développement d'Asie, cette agriculture tient une place de choix. Ainsi, au Vietnam elle produit $80 \%$ des légumes consommés à Hanoï (ETC-Urban Agriculture Programme, 2003). En Afrique de l'Ouest, l'exemple du Benin est typique ; avec «le projet 4 du programme 2, relatif à l'aménagement des bas-fonds, à la promotion des cultures de contre saison notamment les cultures maraîchères et à la création des comités de gestion des bas-fonds » (NOUATIN et BACHABI, 2010). En ce qui concerne la Côte d'Ivoire, cette agriculture et donc le maraîchage intra-urbain reste encore dans l'informel, la précarité et n'est pas prises en compte dans les politiques de gestion des villes. Il $\mathrm{y}$ a certes quelques initiatives telles que le projet d'appui à l'horticulture urbaine et périurbaine, mis en œuvre par le Ministère de l'Agriculture en collaboration avec la $\mathrm{FAO}^{20}$ en 2012. Du matériel de semence d'un montant de 14308152 de francs CFA (SANGAFOWA, 2012) a été remis aux horticulteurs urbains et périurbains par l'Etat ivoirien. Cependant, il ne s'agit que de rares actions ponctuelles. Contrairement à l'agriculture rurale (cultures d'exportations) qui semble plus stratégiques, aucune politique publique clairement définie n'est élaborée en vue de sécuriser les espaces agricoles, encadrer et subventionner les acteurs de l'agriculture urbaine. Dans ces

\footnotetext{
${ }^{20}$ Consciente de l'importance du maraîchage urbain et périurbain, la FAO a initié en 2002 un programme en faveur de l'horticulture urbaine et périurbaine dénommé 'FAO-HUP" dans de nombreux pays en développement.
} 
conditions, les agriculteurs sont à tout moment menacés de déguerpissement dans la mesure où ils ne disposent pas d'espaces délimités et officiellement reconnus pour l'exercice de leur activité. C'est d'ailleurs en cela que BERG (1984) considère le maraîchage périurbain comme «un mode transitoire d'utilisation du foncier ». Les lotissements et autres constructions anarchiques dans les bas-fonds dénotent de la négligence dont fait objet le maraîchage intra-urbain dans les politiques de gestion de la ville de Bouaké.

Ajoutons par ailleurs que cette négligence n'est pas seulement le fait des différents gouvernements qui se sont succédés à la tête de l'Etat ivoirien mais également des collectivités territoriales et des acteurs privés. L'agriculture urbaine n'est pas une priorité et encore moins évoquée dans la planification du développement local des entités décentralisées. Les acteurs privés (opérateurs économiques, sociétés privés) auraient pu booster le développement de cette activité, mais aucune action n'est menée en ce sens. Tout se passe comme si l'agriculture urbaine est la laissée pour compte des politiques de développement local. Pourtant à Bouaké, le maraîchage urbain se présente comme une des potentialités locales à valoriser dans le cadre du développement local intégré. Quoique pourvoyeuse d'emploi et contribuant à la sécurité alimentaire, il reste moins important et moins stratégique par rapport aux cultures pérennes et à l'importation de denrées alimentaires comme le riz. Ainsi, les pouvoirs publics et les collectivités territoriales et autres opérateurs économiques portent leur choix sur ces secteurs qui pour eux sont plus rentables et plus stratégiques.

Retenons pour l'essentiel que malgré son importance en termes de sécurité alimentaire, d'emploi, de protection de l'environnement, le maraîchage urbain reste un secteur négligé dans les politiques publiques. Cette situation le maintient dans l'informel, la précarité et menacé par l'urbanisation anarchique.

\section{Conclusion}

Le maraîchage urbain à Bouaké constitue une activité importante en termes de sécurité alimentaire, d'emploi et de réponse aux problèmes environnementaux. Cependant, ce type d'agriculture fait face à une problématique foncière depuis le déclenchement de la crise militaro-politique de septembre 2002. Il a donc été question, à travers cette étude d'analyser la nouvelle dynamique foncière et la situation agricole en cours dans les basfonds maraîchers de la ville de Bouaké. L'atteinte de cet objectif a nécessité la mobilisation de l'approche qualitative et des techniques de recueil de données; à savoir la recherche documentaire, le guide d'entretien et l'observation directe. Ces données recueillies ont fait l'objet d'une analyse du contenu. 
Comme résultats, les enquêtes ont mis en lumière une nouvelle dynamique foncière en cours dans les bas-fonds intra-urbains à Bouaké ; en raison de l'émergence d'une pluralité d'acteurs ces dernières années. Il en découle la réduction des espaces cultivés, une instabilité et une précarisation du maraîchage urbain. A l'analyse, l'on retient une revalorisation des sols de ces bas-fonds; ce qui donne lieu à la redéfinition de nouvelles règles d'exploitation de ces espaces jadis abandonnés aux seuls maraîchers. A terme, il se pose la problématique de la gouvernance foncière urbaine à travers la juxtaposition de plusieurs droits et donc l'application d'un droit hybride dans la gestion de ces sols hydromorphes à l'intérieur de la ville. Cette situation profitable aux lotissements et constructions anarchiques est consécutive à la négligence même dont fait l'objet le maraîchage urbain dans les politiques publiques et dans la gestion des villes en Côte d'Ivoire.

\section{References:}

1. Anne - Cecile Daniel (2013) - Aperçu de l'agriculture urbaine en Europe et en Amérique du Nord, AgroParistech, 74 p

2. BERG V.D.M. (1984)-Anticipating urban growth in Africa: land use and land values. In the urban fringe of Lusaka, Zambia. Zambian Geographical Association, Occasional Study, $\mathrm{n}^{\circ} 13$.

3. BLAUDIN D. T. C. et al (2009)- La filière agricole au cœur des villes en 2030, AgroParistech, Ministère Français de l'Agriculture et de la Pêche, $60 \mathrm{p}$

4. BOUDON R. (2002) -Théorie du choix rationnel ou l'individualisme méthodologique ? In Sociologie et sociétés, 34 (1) : 9-34

5. CHOUAIBOUB M. M. et SINKONDO M. (2002) - L'Afrique à l'épreuve du développement durable, L'Harmattan, Paris, 297 p

6. CROZIER M. et FRIEDBERG E. (1977) - L'acteur et le système, Editions du Seuil, $500 \mathrm{p}$

7. HESSELING G. (1991)- Les citadins et le droit à la ville : des stratégies diversifiées. In LEBRIS E.et al.-L'appropriation de la terre en Afrique noire : manuel d'analyse, de décision et de gestion foncière, KARTHALA, pp. 203-241

8. ETC - Urban Agriculture Programme (2003). Annotated Bibliography on Urban Agriculture.Leusden, The Netherlands, $804 \mathrm{p}$

9. FLEURY A., DONNADIEU P. (1997). : De l'agriculture périurbaine à l'agriculture urbaine. In Courrier de l'environnement de l'Inra, 31 : 45-61

10. KRA k.V- http://www.fao.org/fsnforum/comment/7919 - Comment faire en sorte que les chaînes de valeur contribuent à améliorer la nutrition? : Le cas de la Côte d'Ivoire en Afrique de l'Ouest. Consulté le 21 mai 2017 
11. LAURENT P.J. (2006) - Décentralisation, services de développement agricole rural et affaires locales. Normes et mode de gestion des ressources (Atelier III). In Le bulletin de l'APAD, n 16, pp31-37

12. MASSIAH G. et TRIBILLON J.F (1988) - Villes en développement, La Découverte, Paris, $320 \mathrm{p}$

13. MATHIJS V. L. and GEMMA V. D. H. (2014) - La gouvernance foncière comme moyen de renforcement de l'Etat au niveau local dans l'Est RDC. In IS Academy Publication occasionnelle 7, 47

14. NOUATIN G. et BACHABI F.X. - URL : http://vertigo.revues.org/10038 - Urbanisation et viabilité de l'activité maraîchère : cas d'une ville à statut particulier au Bénin (Parakou), Volume 10 numéro 2 | septembre 2010, consulté le 22 mai 2017

15. SANGAFOWA M. 2012, http://news.abidjan.net/h/445822.html. Consulté le 22 mai 2017 